Fausto da Silva Gonçalves ${ }^{1}$

Denise Cristina Mos Vaz-Oliani ${ }^{1}$

Gustavo Henrique de OliverRa ${ }^{2}$

ANTÔNIO HélIO OLIANI ${ }^{1}$

Artigo Original

Palavras-chave

Doenças dos anexos

Doenças dos anexos

Doenças dos anexos/ultrassonografia

Telerradiologia

Ultrassonografia

Keywords

Adnexal diseases

Adnexal diseases

Adnexal diseases/ultrasonography

Teleradiology

Ultrasonography

\title{
Avaliação dos parâmetros de imagens de massas anexiais pélvicas em tele-ecografia
}

\author{
Evaluation of image parameters of \\ pelvic adnexal masses in tele-echography
}

\section{Resumo}

OBJETIVO: Avaliar os parâmetros ultrassonográficos relacionados às características morfológicas de massas anexiais pélvicas por meio da obtenção de imagens bidimensionais por um observador presencial, encaminhadas eletronicamente (via tele-ecografia) nos modos estático e dinâmico a observadores não presenciais (à distância) para análises comparativas inter e intraobservadores a fim da validação de uma segunda opinião. MÉTODOS: No período de março a agosto de 2010 foram selecionadas 50 pacientes que apresentavam visualização de massa anexial pélvica no exame ecográfico. Elas foram submetidas ao exame ultrassonográfico por vias abdominal e endovaginal. As imagens foram capturadas nos modos estático e dinâmico, encaminhadas eletronicamente para avaliação de dois examinadores não presenciais e avaliadas de acordo com parâmetros morfológicos ultrassonográficos previamente determinados. RESULTADOS: Os avaliadores não presenciais nas modalidades estática e dinâmica obtiveram concordância quase perfeita para todos os parâmetros morfológicos com valores de Kappa entre 0,6 e 0,8. Não houve diferença entre as modalidades empregadas, exceto para o parâmetro morfológico projeção papilar na modalidade estática, em que a concordância foi quase perfeita $(0,8)$ enquanto na modalidade dinâmica foi substancial $(0,6)$. CONCLUSÕES: Os parâmetros ultrassonográficos das características de massas anexiais pélvicas encaminhadas por tele-ecografia são passíveis de serem validadas para emissão de uma segunda opinião. Não houve diferenças significativas quanto à metodologia empregada na emissão das imagens tele-ecográficas (estática ou dinâmica) na caracterização da composição das massas anexiais pélvicas.

\section{Abstract}

PURPOSE: To evaluate the sonographic parameters related to morphological characteristics of pelvic adnexal masses by obtaining two-dimensional images by an observer presence, forwarded electronically (via tele-ultrasound) in static and dynamic modes observers do not face (distance) for comparative analyzes inter intraobserver and to the validation of a second opinion. METHODS: From March to August 2010 were selected 50 patients with adnexal mass viewing of the pelvic ultrasound. They were subjected to ultrasound for abdominal and transvaginal routes. The images were captured in static and dynamic modes, electronically forwarded for evaluation of non-presence and two examiners evaluated according to morphological ultrasonographic parameters previously determined. RESULTS: The evaluators did not face in static and dynamic modes obtained almost perfect agreement for all morphological parameters with Kappa values between 0.6 and 0.8. There was no difference between the methods employed, except for the morphological parameter papillary projection in the static mode, in which the agreement was almost perfect $(0.8)$ while in dynamic mode was substantial (0.6). CONCLUSIONS: The sonographic parameters of the features of adnexal masses pelvic sent by tele-ultrasound are capable of being validated for issuing a second opinion. There were no significant differences in the methodology used in the issue of tele-ultrasound images (static or dynamic) to characterize the composition of pelvic adnexal masses.

Correspondência

Fausto da Silva Goncalves Faculdade de Medicina de São José do Rio Preto - FaMERP Avenida Brigadeiro Faria Lima, 5.416 - Vila São Pedro

(EPP: 15090-000 São José do Rio Preto (SP), Brasil

Recebido

$14 / 11 / 2012$

Aceito com modificacōes

$04 / 10 / 2013$
Trabalho realizado na Unidade de Imagenologia do Departamento de Obstetrícia e Ginecologia da Faculdade de Medicina São José do Rio Preto - FAMERP - São José do Rio Preto (SP), Brasil.

'Departamento de Obstetrícia e Ginecologia, Faculdade de Medicina de São José do Rio Preto - FAMERP - São José do Rio Preto (SP), Brasil.

2Unidade de Imagenologia do Departamento de Obstetrícia e Ginecologia, Faculdade de Medicina de São José do Rio Preto FAMERP - São José do Rio Preto (SP), Brasil.

Conflito de interesses: não há. 


\section{Introdução}

A ultrassonografia transvaginal (USTV) é o método de imagem escolhido para avaliar os anexos uterinos, especificamente tubas e ovários. Com o aprimoramento da resolução dos equipamentos de ultrassonografia (US), a acuidade diagnóstica aumentou e houve avanço na compreensão dos processos inflamatórios, funcionais, neoplásicos anexiais e, mais especificamente, dos tumores ovarianos ${ }^{1,2}$.

Diversos estudos têm sido realizados para diferenciar as massas anexiais e, assim, reduzir o número de pacientes submetidas a tratamentos inadequados ${ }^{2}$. As estratégias que vem sendo utilizadas são a avaliação das características morfológicas das massas anexiais ultrassonográficas bidimensionais, a dosagem de marcadores tumorais, em especial o CA-125, os modelos com multivariáveis regressivas e, mais recentemente, a avaliação dinâmica das imagens ultrassonográficas (real time $)^{3,4}$.

A despeito dessa pluralidade de metodologias colocadas em prática para caracterizações de massas anexiais pélvicas, todas mostram desempenho inferior à avaliação subjetiva (pattern recogntion) das características morfológicas bidimensionais na predição de malignidade das massas anexiais pélvicas 5 .

A maioria das massas anexiais possui características morfológicas bidimensionais marcantes que permitem predição de sua benignidade ou malignidade e o provável diagnóstico histológico específico. Este fato é altamente relevante para a aplicabilidade em telemedicina (TM), possibilitando a avaliação de imagens anexiais pélvicas bidimensionais capturadas sem a necessidade presencial da paciente ${ }^{6}$.

De acordo com a Organização Mundial da Saúde (OMS), a TM compreende a oferta de serviços ligados aos cuidados de saúde nos casos em que a distância é um fator crítico. Eles são prestados por profissionais da área de saúde usando tecnologias de informação e de comunicação para intercâmbio de informações válidas para diagnósticos, prevenção e tratamento de doenças, além de contínua educação de prestadores de serviços ou para fins de pesquisas e avaliações ${ }^{7,8}$.

O objetivo deste estudo foi a ampliação do acesso das pacientes brasileiras aos exames de ultrassonografia pélvica para a caracterização de massas anexiais, utilizando os parâmetros relacionados às características morfológicas ultrassonográficas, com obtenção de imagens bidimensionais por observador presencial, encaminhadas eletronicamente (via tele-ecografia) no modo estático e dinâmico a observadores não presenciais (à distância), a fim de análises comparativas inter e intraobservadores e validação de uma segunda opinião por TM.

\section{Métodos}

Em conformidade com as normas regulamentadoras de pesquisas em seres humanos este trabalho foi aprovado pelo
Comitê de Ética em Pesquisa da Faculdade de Medicina de São José do Rio Preto (FAMERP), documentado no Processo $n^{\circ} 5907 / 2008$ parecer 403/2008, aprovado em 13 de outubro de 2008.

As pacientes foram selecionadas no ambulatório de ginecologia da FAMERP e encaminhadas à Unidade de Imagenologia do Departamento de Ginecologia e Obstetrícia do Hospital de Base de São José do Rio Preto para exame complementar ultrassonográfico.

No período de março a agosto de 2010, foram selecionadas 50 pacientes com massa anexial ao exame ecográfico.

Os critérios de inclusão foram: ter os exames realizados pelo examinador responsável pelo trabalho, termo de consentimento livre e esclarecido lido e assinado, maioridade, captura de imagem no modo dinâmico e estático, avaliação das características morfológicas pela via endovaginal e visualização de massa anexial pélvica ao exame ultrassonográfico.

Foram excluídas as pacientes que não aceitaram participar do estudo, as com impedimento de realização dos exames ultrassonográficos com sonda endovaginal e as que apresentaram desconforto exacerbado durante a realização do exame.

Os exames ultrassonográficos pélvicos foram realizados por via abdominal e via endovaginal na Unidade de Imagenologia do Departamento de Ginecologia e Obstetrícia do Hospital de Base de São José do Rio Preto. Avaliam-se as massas anexiais de acordo com as suas características morfológicas (Quadro 1) $)^{9}$ e algumas definições e terminologias empregadas pelo International Ovarian Tumor Analysis (IOTA $)^{10}$ com modificações.

Em relação aos critérios de Sassone et al. ${ }^{9}$ as seguintes modificações foram realizadas: os parâmetros morfológicos componente e forma foram incluídos, enquanto a morfologia da parede interna foi excluído. A projeção papilar tornou-se um parâmetro de avaliação morfológico. Por sua vez, septo foi mantido e a classificação em espesso ou fino foi substituída pela classificação em completa ou incompleta.

Neste estudo, o componente foi definido como todo ou parte da composição da massa anexial, podendo ser cístico (composto por um conteúdo líquido, semilíquido ou pastoso), sólido (composto por um conteúdo maciço,

Quadro 1. Características morfológicas das massas anexiais conforme avaliação de Sassone et al. ${ }^{9}$

\begin{tabular}{|c|c|c|c|c|}
\hline $\begin{array}{l}\text { Morfologia da } \\
\text { parede interna }\end{array}$ & Lisa & $\begin{array}{l}\text { Irregular } \\
(<3 \mathrm{~mm})\end{array}$ & Papilas (>3 mm) & $\begin{array}{l}\text { Não se } \\
\text { aplica }\end{array}$ \\
\hline $\begin{array}{l}\text { Espessura da } \\
\text { parede }\end{array}$ & Fina $(<3 \mathrm{~mm})$ & $\begin{array}{c}\text { Espessura }(>3 \\
\mathrm{mm})\end{array}$ & Não se aplica & \\
\hline Septos & Ausentes & Finos $(<3 \mathrm{~mm})$ & Espesso ( $>3 \mathrm{~mm})$ & \\
\hline Ecogenecidade & $\begin{array}{l}\text { Sonulecente } \\
\text { (anecoico) }\end{array}$ & $\begin{array}{c}\text { Baixa } \\
\text { egonecidade }\end{array}$ & $\begin{array}{l}\text { Misto; baixa ecogênica } \\
\text { com núcleo ecogênico }\end{array}$ & $\begin{array}{c}\text { Alta } \\
\text { ecogenicidade }\end{array}$ \\
\hline
\end{tabular}

Traduzido de Sassone et al. ${ }^{9}$ 
sugerindo a presença de tecido miometrial ou estroma ovariano) ou sólido-cístico ${ }^{10}$. A forma foi definida como a representação geométrica e espacial da massa anexial, podendo ser tubular (imagens alongadas de aspecto tubuliforme) e circular (imagem com aspecto arredondado).

A ecotextura de uma massa anexial foi determinada pela capacidade de propagação do som através de um determinado meio e processada em tons de cinza pelo aparelho de ultrassom. Quanto à ecogenicidade, as massas anexiais foram classificadas em: anecogênica (o feixe sonoro se propaga facilmente através do tecido, tendo tonalidade preta no processamento na escala de cinza), hipoecogênico (o feixe sonoro se propaga de modo satisfatório pelo tecido dependendo da sua densidade e da sua impedância acústica, tendo como tonalidade cinza no processamento na escala de cinza) e ecogênico (o feixe sonoro não se propaga pelo tecido, tendo tonalidade branca na escala de cinza $)^{11}$.

Para a análise das características morfológicas das massas anexiais foram utilizadas a via abdominal e, posteriormente, a endovaginal, sendo esta empregada para a avaliação da morfologia.

Nos exames via abdominal, utilizou-se o transdutor multifrequencial, com frequência de $3,5 \mathrm{MHz}$, e nos exames via endovaginal, transdutor multifrequencial de $7 \mathrm{MHz}$. Os aparelhos eram da marca Medison, modelos Accuvix e Sonoace 8000.

Todos os exames ultrassonográficos foram realizados pelo mesmo examinador, sendo este denominado observador presencial. As avaliações de suas características morfológicas foram consideradas referência para os outros avaliadores.

As imagens foram capturadas e armazenadas em CD-ROM nos modos dinâmico e estático, e encaminhadas separadamente e em momentos diferentes para dois observadores não presenciais denominados A e B, ambos especialistas e com ampla experiência nesta área.

A modalidade dinâmica foi obtida percorrendo-se a imagem-alvo nos sentidos látero-lateral e ântero-posterior com captura por meio da função cine save e conversão em arquivos de vídeos com extensão avi (áudio vídeo interleave) pelo programa Sonoview, presente nos aparelhos de ultrassonografia da marca Medison.

Obteve-se a modalidade estática com a captura da imagem fixa ocupando 50 a $75 \%$ do monitor ecográfico, com ênfase para as características morfológicas marcantes de cada imagem e armazenadas por meio do programa Sonoview em arquivos com formato JPEG (joint pictures expert group).

Para avaliar a opinião dos observadores A e B em relação aos parâmetros ultrassonográficos, considerando as imagens estáticas e dinâmicas, efetuou-se uma análise estatística baseada em medidas de concordância Kappa, conforme Landis e Koch ${ }^{12}$, sendo concordância pobre de 0,00 a 0,19 ; razoável de 0,20 a 0,39 , moderada de 0,40 a 0,59 , substancial de 0,60 a 0,79 e quase perfeito de 0,80 a 1,00 .

\section{Resultados}

A amostra foi composta por 50 pacientes com idade entre 20 e 60 anos, sendo a média de 38,2 anos e desvio-padrão de 10,7 anos. Os cincos parâmetros totalizaram 250 análises, sendo a taxa de concordância entre o examinador presencial e os examinadores não presenciais A e B (na modalidade dinâmica e na modalidade estática) de 82,4\% (206 das 250 análises). Eles concordaram em 35 das 50 avaliações para o parâmetro componente; em 49 das 50 avaliações para o forma; em 32 das 50 avaliações para ecotextura; em 43 das 50 para papilas e em 47 das 50 para septações.

Da mesma forma, houve taxa de concordância de 83,2\% (208 avaliações) entre os examinadores não presenciais A e B (na modalidade estática e na modalidade dinâmica). Entre o examinador presencial e o não presencial A (nas modalidades estática e dinâmica), a taxa de concordância foi de $87,6 \%$ (219 avaliações), e entre o examinador presencial e o B (nas duas modalidades), atingiu 89,2\% (223 avaliações).

O grau de concordância, de acordo com o coeficiente Kappa, para a avaliação das características morfológicas das massas anexiais pélvicas entre o examinador presencial e os examinadores não presenciais (em ambas as modalidades) foi substancial para os parâmetros morfológicos — componente (Kappa de 0,71), forma (Kappa de 0,78), ecotextura (Kappa de 0,66), projeções papilares (Kappa 0,66), septações (Kappa de 0,8) (Tabela 1).

Os graus de concordância foram semelhantes entre a modalidade estática e dinâmica de acordo com coeficiente Kappa. Os valores de Kappa na modalidade estática foram de 0,72 para componente, 0,79 para forma, 0,69 para ecotextura, 0,89 para septações e 0,84 para projeções papilares. Na modalidade dinâmica, atingiram 0,71 para componente, 0,74 para forma, 0,62 para ecotextura, 0,76 para septações e 0,60 para projeções papilares.

Para ambas as modalidades, foi obtida concordância substancial para todos os parâmetros morfológicos, exceto projeções papilares e septação. Nestes casos, a modalidade estática obteve concordância superior entre os examinadores não presenciais quando comparada à dinâmica (para projeção papilar Kappa, =0,84xKappa $\mathrm{MD}=0,6$; para septação Kappa ME =0,89xKappa MD =0,76) (Tabela 2).

Tabela 1. Grau de concordância (Kappa) na análise morfológica das massas anexiais entre os examinadores

\begin{tabular}{lcc}
\hline Parâmetros & Valores de Kappa & IC95\% \\
\hline Componente & 0,7 & $0,58-0,75$ \\
Forma & 0,7 & $0,51-0,73$ \\
Ecotextura & 0,6 & $0,59-0,43$ \\
Septações & 0,8 & $0,70-0,85$ \\
Projeções papilares & 0,6 & $0,56-0,76$ \\
\hline
\end{tabular}

Valor $p<0,001$ 
Tabela 2. Medida de concordância (Kappa) na análise das características morfológicas das massas anexiais entre a modalidade dinâmica e a modalidade estática

\begin{tabular}{lcc}
\hline \multirow{2}{*}{ Parâmetros } & \multicolumn{2}{c}{ Valores de Kappa } \\
\cline { 2 - 3 } & 0,72 & MD \\
\hline Componente & 0,79 & 0,71 \\
Forma & 0,69 & 0,74 \\
Ecotexfura & 0,89 & 0,62 \\
Septações & 0,84 & 0,76 \\
Projeções papilares & 0,60 \\
\hline ME: modalidade estática - examinador presencial x examinadores não presenciais \\
(A e B); MD: modalidade dinâmica - examinador presencial x examinadores \\
não presenciais (A e B).
\end{tabular}

\section{Discussão}

A maioria das massas anexiais pélvicas apresentam parâmetros ultrassonográficos típicos que possibilitam a predição do seu diagnóstico por um examinador experiente e com boa acurácia ${ }^{13,14}$. Estes parâmetros são válidos para as massas anexiais benignas, como os teratomas, endometriomas, corpos lúteos, cistos hemorrágicos, hidrossalpinges, cistos paraovarianos e alguns tumores sólidos (fibromas, tecomas e tumor de Brenner). Da mesma maneira, determinados padrões morfológicos ultrassonográficos estão intimamente relacionados a processos malignos, entre eles massas anexiais irregulares, presença de componente sólido e/ou de projeções sólidas papilares ${ }^{14,15}$.

Dados da IOTA demonstram que aproximadamente 90\% das massas anexiais são passíveis de serem diferenciadas entre malignas ou benignas com o uso da avaliação subjetiva das características morfológicas (pattern recognition). Além disso, observa-se sensibilidade de cerca de $85 \%$ na predição do provável diagnóstico histológico ${ }^{14}$.

É importante salientar que a habilidade para a realização do correto diagnóstico específico de massas anexiais pélvicas pode variar entre os estudos, principalmente em decorrência de amostragem utilizada em cada um deles e da experiência dos examinadores ${ }^{12,15}$.
Neste trabalho não houve diferenças quanto à metodologia empregada (estática ou dinâmica) na caracterização da composição das massas anexiais pelos observadores A e B. O resultado diverge dos encontrados por Van Holsbeke et al. ${ }^{16}$, no qual o diagnóstico de massas anexiais utilizando imagens em tempo real (acurácia de 89\%) demonstrou ser mais preciso do que os realizados com o uso de imagens estáticas (acurácia de 85\%).

Tal fato se deve provavelmente aos vídeos curtos capturados na modalidade dinâmica, que, neste estudo, capturou as imagens em movimento utilizando a função cine save. Presente nos aparelhos utilizados, ela permite a gravação de aproximadamente 250 quadros, o que corresponde a cerca de 10 segundos de gravação.

Os parâmetros morfológicos ultrassonográficos projeções papilares e septações obtiveram grau de concordância moderada, porém divergente entre as modalidades dinâmica e estática considerando os examinadores não presenciais. A estática demonstrou superioridade na avaliação destes parâmetros. Assim, na presença de projeções papilares e septações, o ideal seria o envio tanto de imagens estáticas quanto dinâmicas ${ }^{17}$.

É importante salientar que apesar da boa concordância na análise da composição das massas anexiais presente neste estudo, não se pode afirmar que também haveria boa concordância nas impressões diagnósticas, embora estudos prévios tenham demonstrado isso. Não havia disponibilidade dos resultados dos estudos histológicos (padrão ouro) de todas as pacientes incluídas no trabalho devido ao predomínio de massas anexiais com forte indicação de benignidade, para as quais não se justificaria uma atitude intervencionista.

Conclui-se, então, que os parâmetros ultrassonográficos das características morfológicas de massas anexiais pélvicas encaminhadas por tele-ecografia são passíveis de serem validadas para a emissão de uma segunda opinião e que não houve diferenças significativas quanto à metodologia empregada na emissão das imagens tele-ecográficas (estática ou dinâmica) na caracterização da composição das massas anexiais pélvicas.

\section{Referências}

1. American College of Obstetricians and Gynecologists. ACOG. Management of adnexal masses. Obstet Gynecol. 2007;1 10(1):201-14.

2. Alcázar JL, Díaz L, Flórez P, Guerriero S, Jurado M. Intensive training program for ultrasound diagnosis of adnexal masses: protocol and preliminary results. Ultrasound Obstet Gynecol. 2013;42(2):218-23.

3. Valentin L, Hagen B, Tingulstad S, Eik-Nes S. Comparison of 'pattern recognition' and logistic regression models for discrimination between benign and malignant pelvic masses: a prospective cross validation. Ultrasound Obstet Gynecol. 2001;18(4):357-65.
4. Lerner JP, Timor-Tritsch IE, Federman A, Abramovich G. Transvaginal ultrasonographic characterization of ovarian masses with an improved, weighted scoring system. Am J Obstet Gynecol. 1994;170(1 Pt 1):81-5.

5. Alonzo TA. Standards for reporting prognostic tumor marker studies. J Clin Oncol. 2005;23(36):9053-4.

6. Timmerman D, Van Calster B, Testa AC, Guerriero S, Fischerova $D$, Lissoni $A A$, et al. Ovarian cancer prediction in adnexal masses using ultrasound-based logistic regression models: a temporal and external validation study by the IOTA group. Ultrasound Obstet Gynecol. 2010;36(2):226-34. 
7. World Health Organization [Internet]. Diagnostic imaging. Geneva: WHO; 2011 . [cited 2011 Nov 26]. Available from: <http:// www.who.int/diagnostic_imaging/en/>

8. Ferlin RM, Oliani AH, Vaz-Oliani DM, Ferreira AC, Tristão EG, Mauad Filho F. Tele-ecografia em tempo real: um estudo piloto. Rev Imagem (Online) [Internet]. 2010];32(1/2): 1-6 [citado 2011 Nov 26. Disponível em: <http://www.spr.org.br/files/public/ magazine/public_223/01-06.pdf>

9. Sassone AM, Timor-Tritsch IE, Artner A, Westhoff C, Warren WB. Transvaginal sonographic characterization of ovarian disease: evaluation of a new scoring system to predict ovarian malignancy. Obstet Ginecol. 1991:78(1):70-6.

10. Timmerman D, Valentin L, Bourne TH, Collins WP, Verrelst $H$, Vergote I. Terms, definitions and measurements to describe the sonographic features of adnexal tumors: a consensus opinion from the International Ovarian Tumor Analysis (IOTA) Group. Ultrasound Obstet Gynecol. 2000; 16(5):500-5.

11. Francisco LD, Francisco Neto M, Pastore AR. Abordagens das massas pélvicas ginecológicas. In: Pastore AR, Cerri GG. Ultra-sonografia, Obstetrícia e Ginecologia. São Paulo: Sarvier; 1997. p. 573-83.
12. Landis JR, Koch GG. The measurement of observer agreement for categorical data. Biometrics. 1977;33(1):159-74.

13. Valentin L. Use of morphology to characterize and manage common adnexal masses. Best Pract Res Clin Obstet Gynaecol. 2004; 18(1):71-89.

14. Sokalska A, Timmerman D, Testa AC, Van Holsbeke C, Lissoni AA, Leone FP, et al. Diagnostic accuracy of transvaginal ultrasound examination for assigning a specific diagnosis to adnexal masses. Ultrasound Obstet Gynecol. 2009;34(4):462-70.

15. Faschingbauer F, Benz M, Häberle L, Goecke TW, Beckmann MW, Renner $S$, et al. Subjective assessment of ovarian masses using pattern recognition: the impact of experience on diagnostic performance and interobserver variability. Arch Gynecol Obstet. 2012;285(6): 1663-9.

16. Van Holsbeke C, Yazbek J, Holland TK, Daemen A, De Moor $B$, Testa $A C$, et al. Real-time ultrasound vs. evaluation of static in the preoperative assessment of adnexal masses. Ultrasound Obstet Gynecol. 2008;32(6):828-31.

17. Jain KA. Prospective evaluation of adnexal masses with endovaginal greyscale and duplex and color Doopler US: correlation with pathologic findings. Radiology. 1994;191(1):63-7. 